\title{
A triboelectric nanogenerator based on cosmetic fixing powder for mechanical energy harvesting
}

\author{
Kequan Xia', Yue Chi ${ }^{1}$, Jiangming Fu' ${ }^{1}$ Zhiyuan Zhu' ${ }^{1}$, Hongze Zhang ${ }^{2}$, Chaolin $\mathrm{Du}^{1}$ and Zhiwei Xu ${ }^{1}$
}

\begin{abstract}
In this work, we use commercial powder particulates (a cosmetic fixing powder) as triboelectric materials for constructing a triboelectric nanogenerator (CFP-TENG). Through finger pressing, the CFP-TENG generated approximate open-circuit voltage, short-circuit current, and maximum power density values of $1141 \mathrm{~V}, 521 \mu \mathrm{A}$, and $570.96 \mu \mathrm{W} / \mathrm{cm}^{2}$, respectively. Thirty-seven commercial blue LEDs can be easily lit up by the CFP-TENG. Moreover, this TENG, which was designed as a novel palette structure for harvesting mechanical energy from bicycle motion, serves as a self-powered bicycle speed sensor. In addition, the cosmetic fixing powder can be used as an effective material to enhance the triboelectric property of skin. This study provides an effective method for developing a cost-effective TENG without the use of complex surface micro-/nano-texturing.
\end{abstract}

\section{Introduction}

The global energy crisis is becoming increasingly severe with the fast-paced growth of the global economy ${ }^{1-3}$. Moreover, with the continued acceleration of human civilization, exhaustion of fossil energy in the first half of the twenty-first century is expected ${ }^{4,5}$. Renewable energy for electricity generation has been considered a means of solving the energy crisis ${ }^{6-10}$. Solar energy, tidal energy, mechanical motion, and thermal changes are all considered potential forms of energy that are convertible into electrical energy in the environment. Among these, mechanical energy is the most extensively distributed type, as it occurs in diverse forms ${ }^{11,12}$. Daily activities such as walking, running, cycling, and even tiny facial expressions (for example, smiling and crying) are accompanied by mechanical distortions, suggesting that the human body constitutes a pivotal means of mechanical power generation ${ }^{13,14}$. However, it is difficult to apply traditional electromagnetic induction generators for harvesting

Correspondence: Zhiyuan Zhu (zyzhu@zju.edu.cn) or

Zhiwei Xu (xuzw@zju.edu.cn)

${ }^{1}$ Ocean College, Zhejiang University, 316021 Zhejiang, China

${ }^{2}$ Nanjing Electronic Devices Institute, 210016 Jiangsu, China mechanical energy from the surrounding environment (human daily activities, etc.).

In 2012, Prof. Zhonglin Wang (Georgia Institute of Technology) proposed the first-ever triboelectric nanogenerator (TENG), which converts mechanical energy from the surrounding environment into electrical energy on the basis of the triboelectric effect ${ }^{15-25}$. During the past few years, the TENG has received considerable attention and has been widely applied to multidisciplinary fields owing to its outstanding output performance, sustainable power output, and ease of integration ${ }^{26-34}$. Several materials (for example, PDMS, Kapton, $\mathrm{Al}$, and Teflon, each with its own merits and demerits) have been applied to the construction of TENGs ${ }^{35-41}$. Although silica-based powder is also used as one of the triboelectric pair $^{42}$, the output voltage is relatively low $(\sim 11 \mathrm{~V})$. The triboelectric substances proposed for TENG construction should (ideally) be low cost, yield rapidly self-forming films, be processable without the need for cumbersome micro-/nano-processing, and (if possible) be readily available.

In this work, we propose a novel concept of a powder TENG that uses powder particulates (for example,

\section{(c) The Author(s) 2019}

(c) (i) Open Access This article is licensed under a Creative Commons Attribution 4.0 International License, which permits use, sharing, adaptation, distribution and reproduction cc) in any medium or format, as long as you give appropriate credit to the original author(s) and the source, provide a link to the Creative Commons license, and indicate if changes were made. The images or other third party material in this article are included in the article's Creative Commons license, unless indicated otherwise in a credit line to the material. If material is not included in the article's Creative Commons license and your intended use is not permitted by statutory regulation or exceeds the permitted use, you will need to obtain permission directly from the copyright holder. To view a copy of this license, visit http://creativecommons.org/licenses/by/4.0/. 


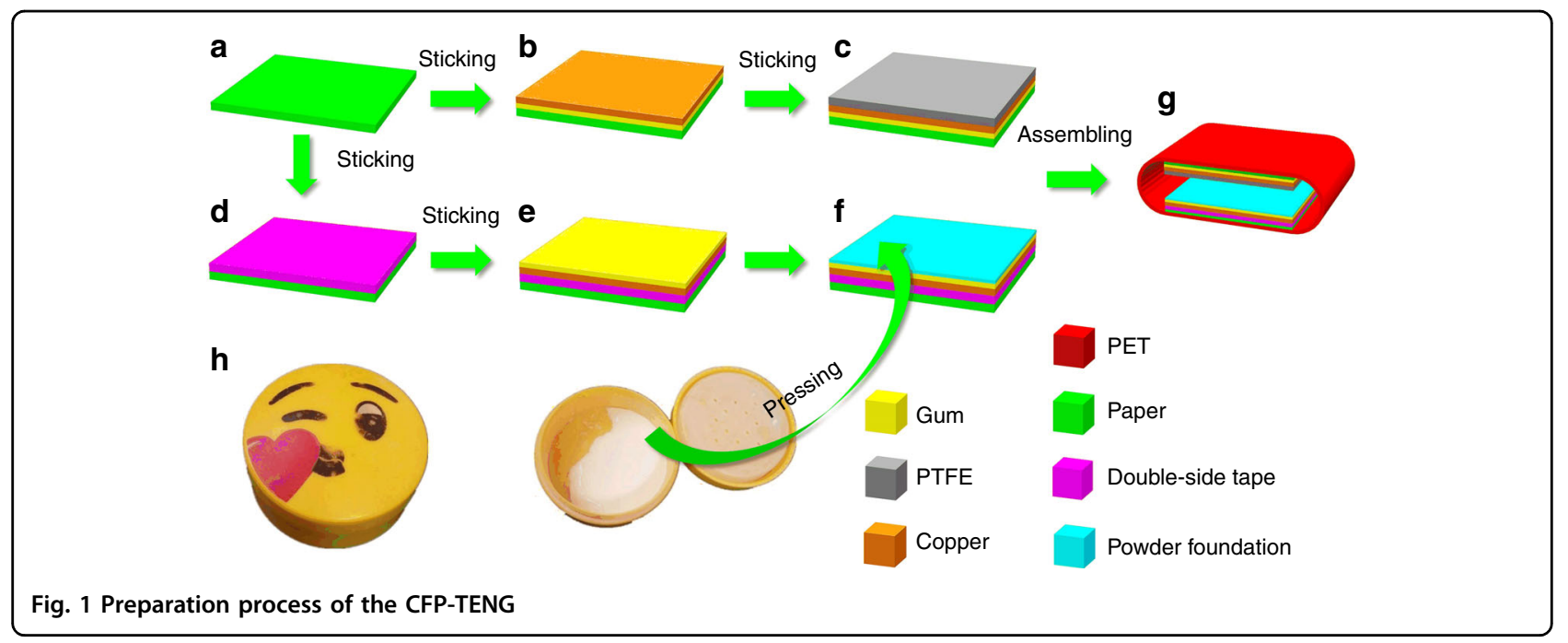

cosmetic fixing powder) as triboelectric materials for fabricating a triboelectric nanogenerator (CFP-TENG). This fabrication, which is realized without the use of surface micro-/nano-texturing techniques that rely on expensive equipment and complex technology, may drive the large-scale application of TENGs in multidisciplinary fields. In our work, this powder and Teflon tape were used as the triboelectric pair. The CFP-TENG can, through finger pressing, produce approximate open-circuit voltage, short-circuit current, and power density values of $1141 \mathrm{~V}, 521 \mu \mathrm{A}$, and $570.96 \mu \mathrm{W} / \mathrm{cm}^{2}$, respectively. Thirtyseven commercial blue LEDs, which were assembled into the word "ZJU", could be lit up by the CFP-TENG. In addition, this TENG, which was designed as a novel palette structure to harvest mechanical energy from bicycle motion, serves as a self-powered speed sensor. In addition, the cosmetic fixing powder can be used as an effective material to enhance the triboelectric property of skin, which is significant for the development of a humanbased TENG.

\section{Results}

The design and fabrication process of the CFP-TENG device is schematically presented in Fig. 1. First, a sheet of paper was cut into two pieces of paper substrate (size: $3 \mathrm{~cm} \times 3 \mathrm{~cm}$ ), as shown in Fig. 1a. A piece of copper tape $(3 \mathrm{~cm} \times 3 \mathrm{~cm})$ was then pasted onto the paper surface (see Fig. 1b). Subsequently, Teflon tape was pasted onto the surface of the copper tape, thereby forming the top section of the TENG, as shown in Fig. 1c. Another paper substrate was obtained by pasting the double-sided tape onto the paper substrate surface, as shown in Fig. 1d, and then attaching the copper to the surface of the tape. Afterward, the cosmetic fixing powder was applied onto the glue side of the copper tape. Excess powder was then
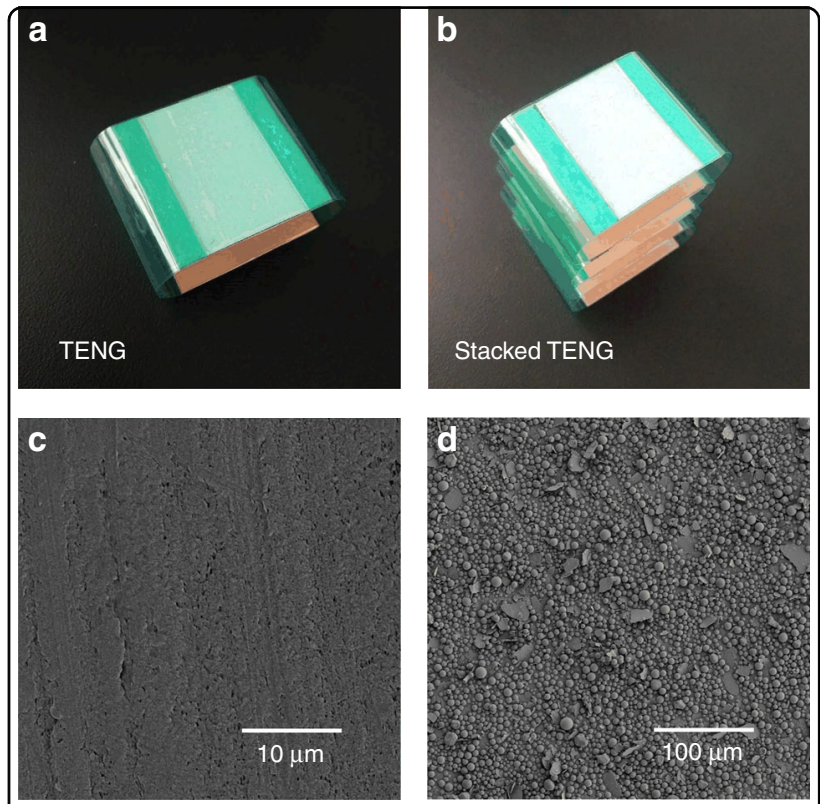

Fig. 2 Images of the device and triboelectronic surfaces. Photographs of the $\mathbf{a}$ CFP-TENG unit and $\mathbf{b}$ stacked CFP-TENG. SEM image of the $\mathbf{c}$ Teflon tape surface and $\mathbf{d}$ cosmetic fixing powder layer surface

removed by air blowing, thereby forming the bottom section of the TENG (see Fig. 1e, f). A piece of Polyethylene terephthalate (PET) film served as the supporting structure for assembly of the TENG, as illustrated in Fig. 1g.

Figure 2a, b shows photographs of the CFP-TENG (one unit) and the stacked CFP-TENG. Representative scanning electron microscopy images showing the surface of the Teflon tape and cosmetic fixing powder layer are 
presented in Fig. 2c, d. A mechanical vibrator was used to activate the CFP-TENG. The top and bottom of the TENG were affixed to the pressing surface of the vibrator and a flat panel, respectively. The respective electrical output signals were then measured with a digital oscilloscope.

The working principle of the CFP-TENG is schematically illustrated in Fig. 3. When Teflon tape is in contact with cosmetic fixing powder, electron transfer from the powder layer to the tape (Fig. 3a). A latent electrical difference is expected with separation of the tape from the powder. This type of latent difference drives the electron flow via the external loads from the top $\mathrm{Cu}$ foil to the bottom $\mathrm{Cu}$ foil. This flow screens the positive triboelectric charges available on the paper, thereby producing an output current signal (Fig. 3b). A new electrical equilibrium is expected at the largest separation in a complete contact-separation cycle (Fig. 3c). Subsequent to this equilibrium, contact between the Teflon tape and the cosmetic fixing powder layer is re-established, leading to an imbalance between the stimulated charges on the $\mathrm{Cu}$ electrodes. This imbalance results in electron flow back to the top $\mathrm{Cu}$ foil, thereby producing a reversed output current signal (Fig. 3d). When full contact between the tape and paper is re-established, the CFP-TENG reverts to its initial position, as shown in Fig. 3a. To understand this mechanism, the latent distribution is simulated with COMSOL multiphysics software. An open-circuit a

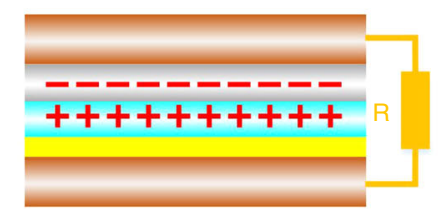

Approaching

d

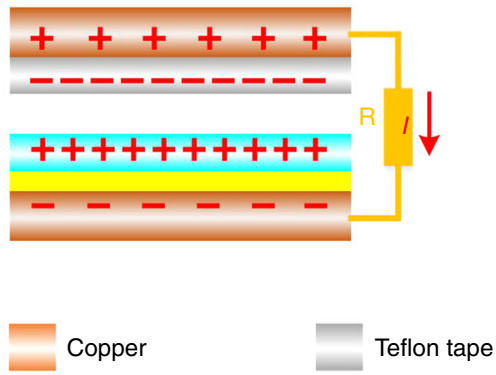

b

Releasing

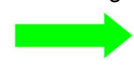

Pressing

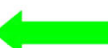

Gum

C

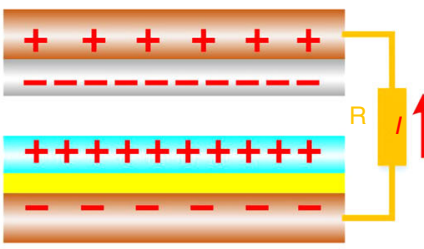

Separating

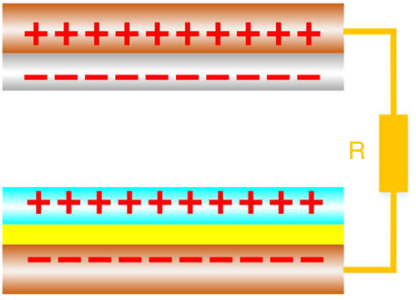

Cosmetic fixing powder e

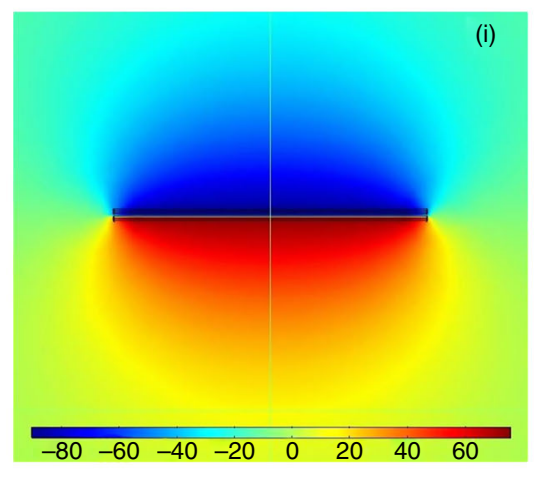

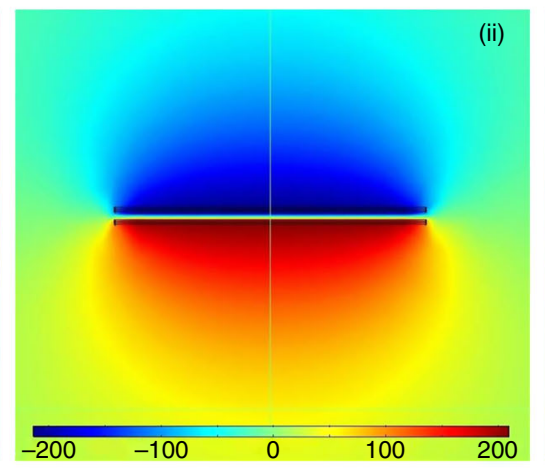

(ii)

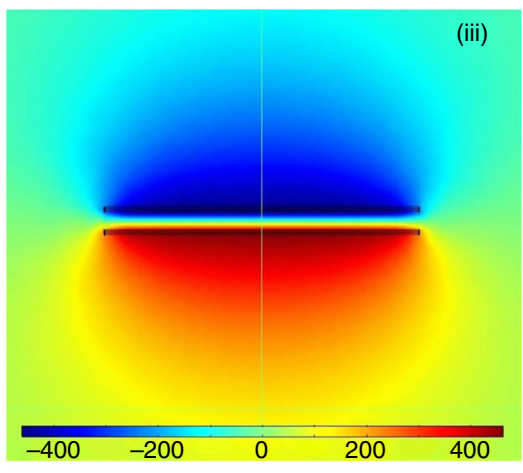

Fig. 3 Schematic and modeling analysis of the device. a-d Working principle of the CFP-TENG. e Numerical calculations (as assessed with the help of COMSOL) of the potential distribution across TENG electrodes, at each step (i-iii), subjected to open-circuit conditions (the voltage unit is volts) 
scenario is considered for the three positions of the oneunit CFP-TENG (see Fig. 3e).

An adjustable resistor ( $100 \mathrm{k} \Omega$ to $1 \mathrm{G} \Omega$ ) was used as the external load of the fabricated TENGs, and the electronic voltage on the resistor was measured. The output current can be derived from the measured voltage and the total resistance. The voltage (under a $1 \mathrm{G} \Omega$ load) and current (under a $100 \mathrm{k} \Omega$ load) of the fabricated CFP-TENG are $498 \mathrm{~V}$ and $30.8 \mu \mathrm{A}$, respectively (see Fig. 4a, b), when triggered by a mechanical vibrator (amplitude: $\sim 5 \mathrm{~cm}$, frequency: $5 \mathrm{~Hz}$ ). For the reverse connection of the oscilloscope to the CFP-TENG, the output voltage and current signals are inverted (as shown in Fig. 4d, e), which indicates that the signals were generated by the CFPTENG. Considering the $200 \mathrm{M} \Omega$ probe of the oscilloscope, the total resistance can be calculated by $R_{\mathrm{t}}=$ $R \times 200 \mathrm{M} \Omega /(R+200 \mathrm{M} \Omega)$, and the corresponding output performance of CFP-TENG is further investigated by evaluating the output power associated with the voltage and current occurring at equivalent total resistances ranging from $99.95 \mathrm{k} \Omega$ to $166.67 \mathrm{M} \Omega$ (see Fig. 4c). With increasing total resistance, the current amplitude decreases owing to a loss of resistance, whereas the voltage increases. In addition, an approximate maximum power density value of $369.17 \mu \mathrm{W} / \mathrm{cm}^{2}$ corresponding to a total resistance of $\sim 26 \mathrm{M} \Omega$ (see Fig. $4 \mathrm{f}$ ) is obtained. In addition, the output performances with and without cosmetic fixing powder are compared in the Supporting Information.

The electronic output performance of the CFP-TENG, based on contact separation, exhibits a high dependence on the contact frequency, separation distance, and device size. Figure $5 \mathrm{a}, \mathrm{b}$ shows the dependence of the $V_{\mathrm{OC}}$ and $I_{\mathrm{SC}}$ values of CFP-TENG on contact frequency for a given device size and separation distance of $3 \mathrm{~cm} \times 3 \mathrm{~cm}$ and $5 \mathrm{~mm}$, respectively. Moreover, the output voltage increases from 325 to $515 \mathrm{~V}$ when the contact frequency increases from 2 to $7 \mathrm{~Hz}$. Nonetheless, the $I_{\mathrm{SC}}$ increases continuously from 19.5 to $32.8 \mu \mathrm{A}$ when the contact frequency increases from 2 to $7 \mathrm{~Hz}$. The increase in the $I_{\mathrm{SC}}$ and $V_{\mathrm{OC}}$ values is attributed to the rapid induction and charge transfer resulting from the elevated frequency contact. Figure $5 \mathrm{c}, \mathrm{d}$ shows the $V_{\mathrm{OC}}$ and the $I_{\mathrm{SC}}$ values of the $(3 \mathrm{~cm} \times 3 \mathrm{~cm}) \mathrm{CFP}$ TENG as a function of the separation distance. Furthermore, with increasing separation distance, the $V_{\mathrm{OC}}$ increases slowly and then saturates, while the $I_{\mathrm{SC}}$ increases continuously. The $V_{\mathrm{OC}}$ and $I_{\mathrm{SC}}$ values corresponding to a contact frequency and separation distance of $5 \mathrm{~Hz}$ and $5 \mathrm{~mm}$, respectively, of TENGs with varied sizes are shown in Fig. 5e, f. As the figure shows, $V_{\mathrm{OC}}$ and $I_{\mathrm{SC}}$ increase with increasing device size. The substantial enhancement in the output is attributed to the augmented contact region. Detailed discussions of the impact of the separation displacement and frequency on the electrical performance are provided in the Supporting Information.

Furthermore, a stacked CFP-TENG is designed and manufactured to improve the output efficiency. The $I_{\mathrm{SC}}$ values of the stacked CFP-TENGs with 2, 3, and 4 units are 51, 72, and $98 \mu \mathrm{A}$, respectively (see Fig. $5 \mathrm{~g}$ ). The results revealed that the output current increases with increasing number of units. Similarly, the stability of the CFP-TENG is evaluated from the findings presented in
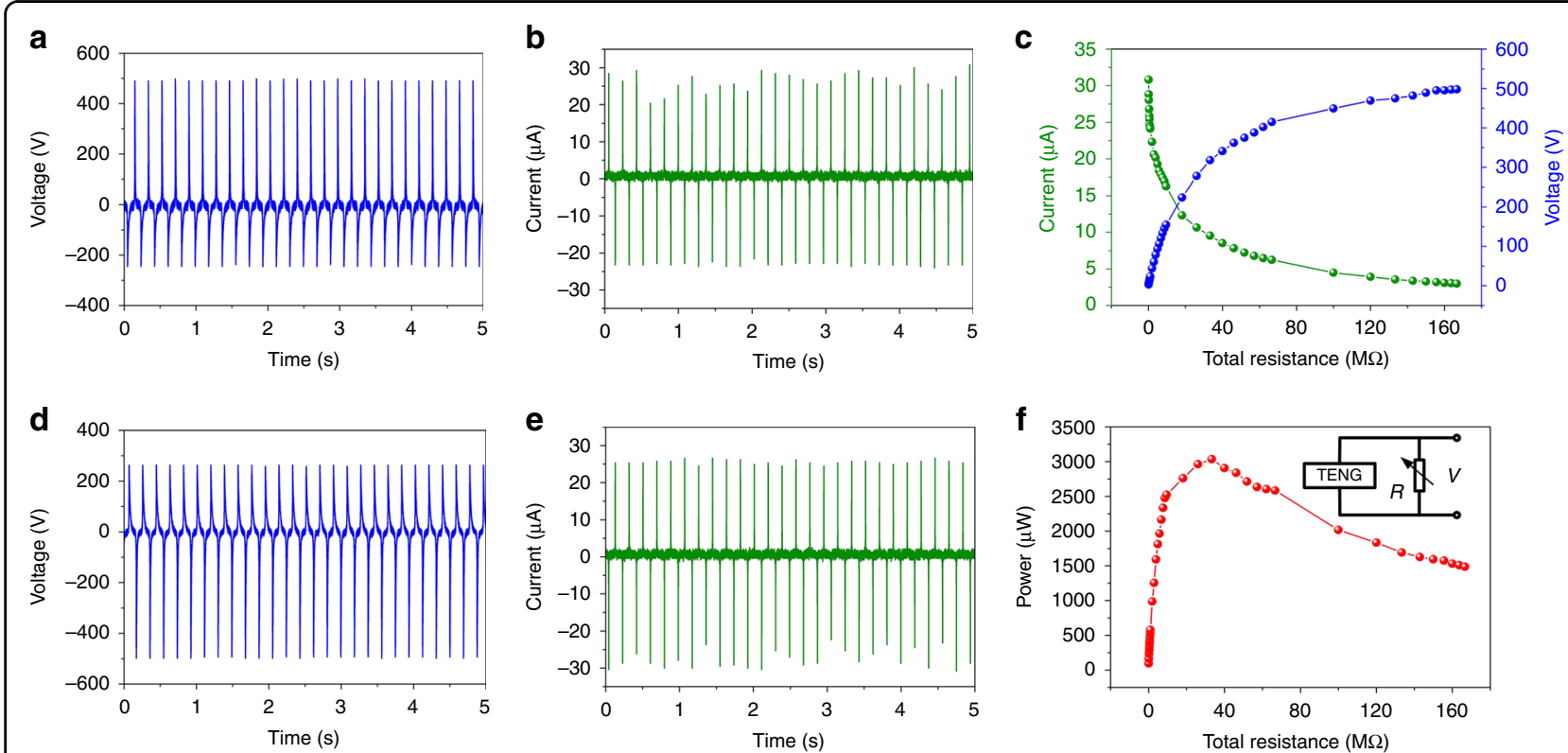

Fig. 4 Electrical output of the device. Output voltage and current of the CFP-TENG calculated using the $\mathbf{a}, \mathbf{b}$ forward connection and $\mathbf{d}$, e reverse connection. Dependence of $\mathbf{c}$ the output voltage and current on the total resistance and $\mathbf{f}$ the power density on the total resistance 

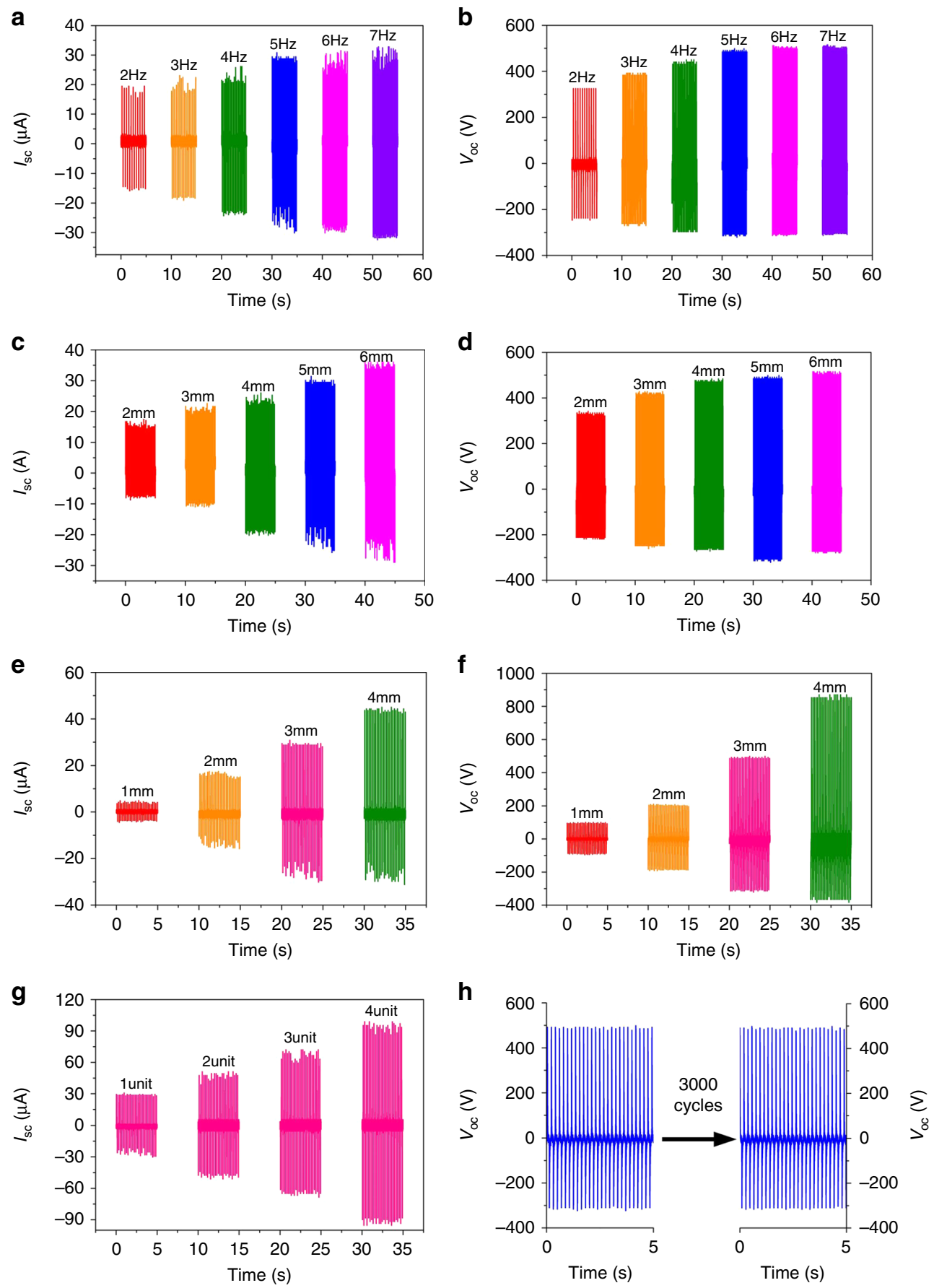

Fig. 5 Performance and stability of the device. Approximate values of $V_{O C}$ and $I_{S C}$ at various a, $\mathbf{b}$ frequencies (CFP-TENG size: $3 \mathrm{~cm} \times 3 \mathrm{~cm}$, maximum separation distance: $5 \mathrm{~mm}$ ), $\mathbf{c}$, d separation distances (mechanical frequency: $5 \mathrm{~Hz}$, CFP-TENG size: $3 \mathrm{~cm} \times 3 \mathrm{~cm}$ ), and e, f CFP-TENG sizes (working frequency: $5 \mathrm{~Hz}$, separation distance of the TENG: $5 \mathrm{~mm}$. g Output current of the CFP-TENG with various unit numbers. $\mathbf{h}$ Stability verification of the fabricated CFP-TENG via 3000 cycles of continuous operation

Fig. 5h. In accordance with the experimental results, a stable output voltage from the CFP-TENG is realized even after a 3000-cycle external force test.

According to a previous study ${ }^{43}$, the human body produces hundreds of watts through body movements.
Therefore, harvesting low-frequency power generated by human physical movements is considered a potential means of realizing self-powered wearable electronics. In this work, we demonstrate that a CFP-TENG $(5 \mathrm{~cm} \times$ $5 \mathrm{~cm}$ ) can be activated via finger pressing (see Fig. $6 \mathrm{f}$ ). As 
shown in Fig. 6a, b, $I_{\mathrm{SC}}$ and $V_{\mathrm{OC}}$ reach approximate values of $521 \mu \mathrm{A}$ and $1141 \mathrm{~V}$, respectively. When a match load of $30 \mathrm{M} \Omega$ (equivalent total resistance: 26.09 $\mathrm{M} \Omega$ ) is connected to the CFP-TENG, the maximum output power, corresponding to an output voltage of $610 \mathrm{~V}$ and current of $23.4 \mu \mathrm{A}$, is reached (see Fig. 6c, d). The corresponding maximum output power $(12.383 \mathrm{~mW})$ and power density $\left(570.96 \mu \mathrm{W} / \mathrm{cm}^{2}\right)$ are adequate for various low-energy-consumption microelectronic gadgets. We also performed tests aimed at determining the charging potential of the fabricated CFP-TENG connected to a $1 \mathrm{nF}$ capacitor by means of a full-wave rectifier bridge. The results revealed that $\sim 85 \mathrm{nC}$ of charge is transferred in one cycle.

\section{Discussion}

To demonstrate the potential of the CFP-TENG as an energy source, a $3 \mathrm{~cm} \times 3 \mathrm{~cm}$ CFP-TENG was linked to 37 commercial blue LEDs in series (as presented in Fig. $7 \mathrm{a}-\mathrm{c})$. The results revealed that the assembly of these LEDs forming the word "ZJU" could be easily lit up by the TENG.

In addition, we propose a novel palette structure for harvesting the mechanical power associated with bicycle movement (see Fig. 8a). Moreover, the proposed structure can also monitor the bicycle speed. The corresponding output voltage of the device (under a $1 \mathrm{G} \Omega$ load) can reach 13.1, 15.7, and $32.5 \mathrm{~V}$ for 50, 100, and 350 r.p.m., respectively, as shown in Fig. $8 \mathrm{~b}-\mathrm{d}$. In addition, the
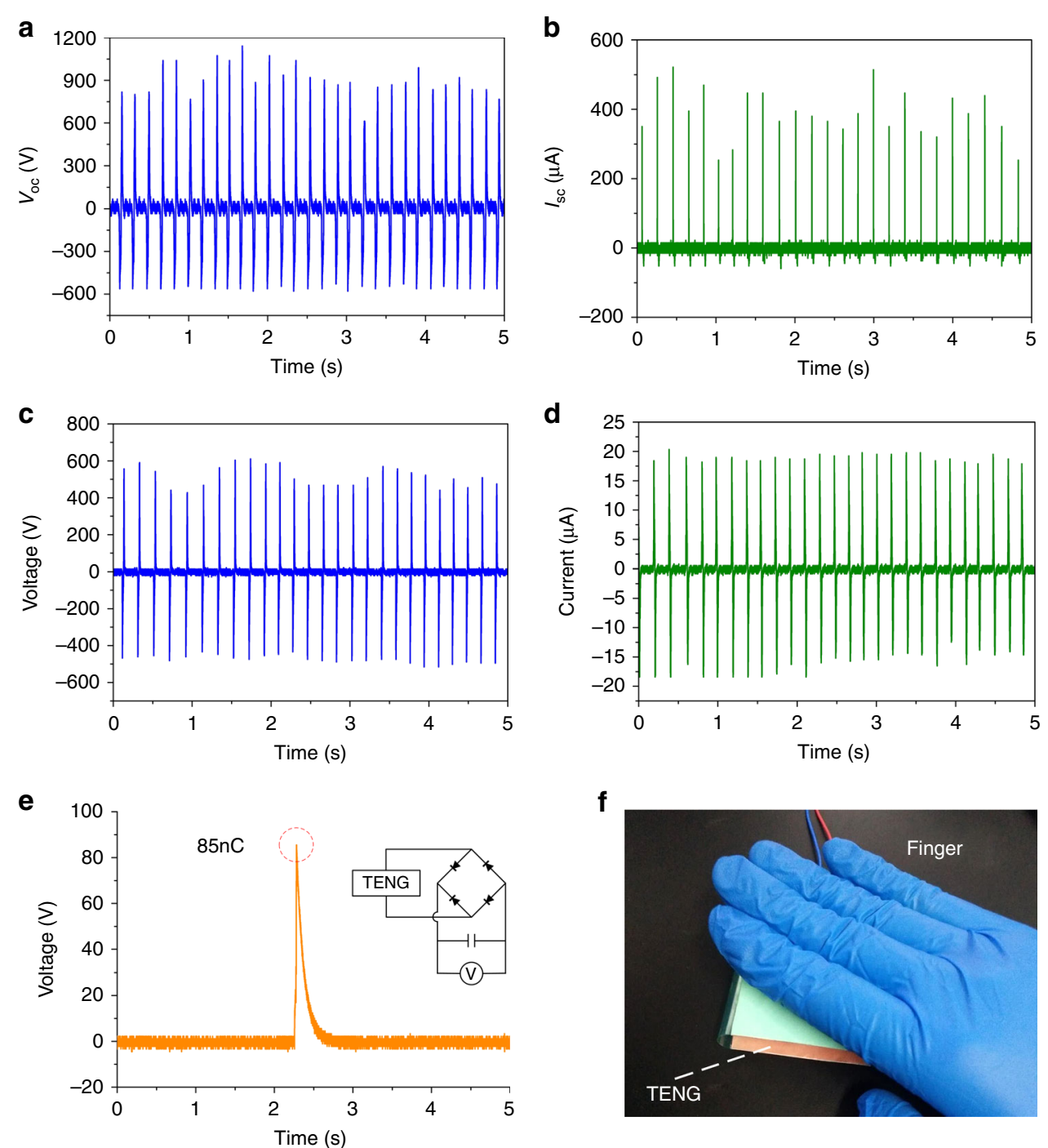

Fig. 6 Electrical characteristics of the CFP-TENG powered by physical movement (finger pressing). Approximate $\mathbf{a} V_{O C}$ and $\mathbf{b} /_{S C}$ values of the CFP-TENG; c output voltage and $\mathbf{d}$ current corresponding to a match load of $30 \mathrm{M} \Omega$ (equivalent total resistance: $26.09 \mathrm{M} \Omega$ ); e voltage of a $1 \mathrm{nF}$ capacitor linked to the CFP-TENG by means of a full-wave rectifier bridge, indicating the quantity of charge transferred in one cycle; and $\mathbf{f}$ a CFP-TENG triggered by finger pressing 
output voltages subjected to different speeds are shown in Fig. 8e. It is observed that the output voltage is approximately linear to the speed.

Furthermore, we proposed a new application of a powderenhanced skin-based TENG. In detail, when the skin is made as the triboelectric layer, the output voltage can reach
$137 \mathrm{~V}$, as shown in Fig. 9a. When the skin is modified by cosmetic fixing powder, the output voltage can reach $274 \mathrm{~V}$, as shown in Fig. 9b. According to the results, the cosmetic fixing powder can be used as an effective material to enhance the triboelectric property of skin, which is significant for the development of a human-based TENG.

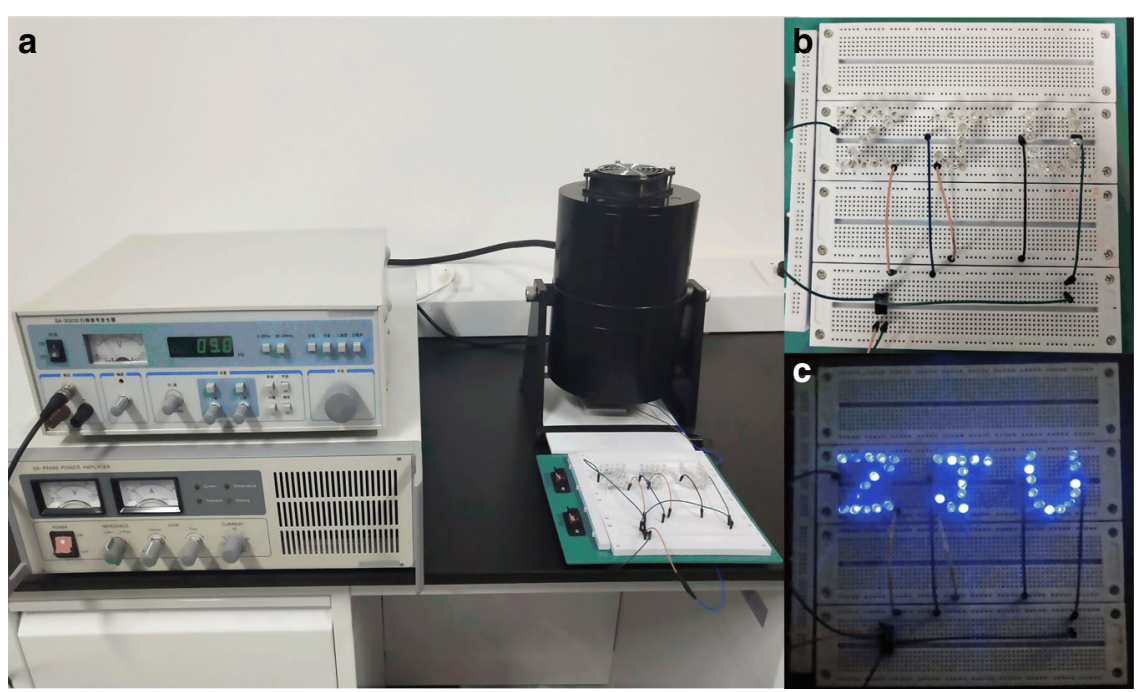

Fig. 7 Driving capability of the device. a A CFP-TENG connected in series with 37 commercial blue LEDs (the inset shows a $3 \mathrm{~cm} \times 3 \mathrm{~cm}$ TENG); b, c 37 commercial blue LEDs powered by finger pressing of the CFP-TENG
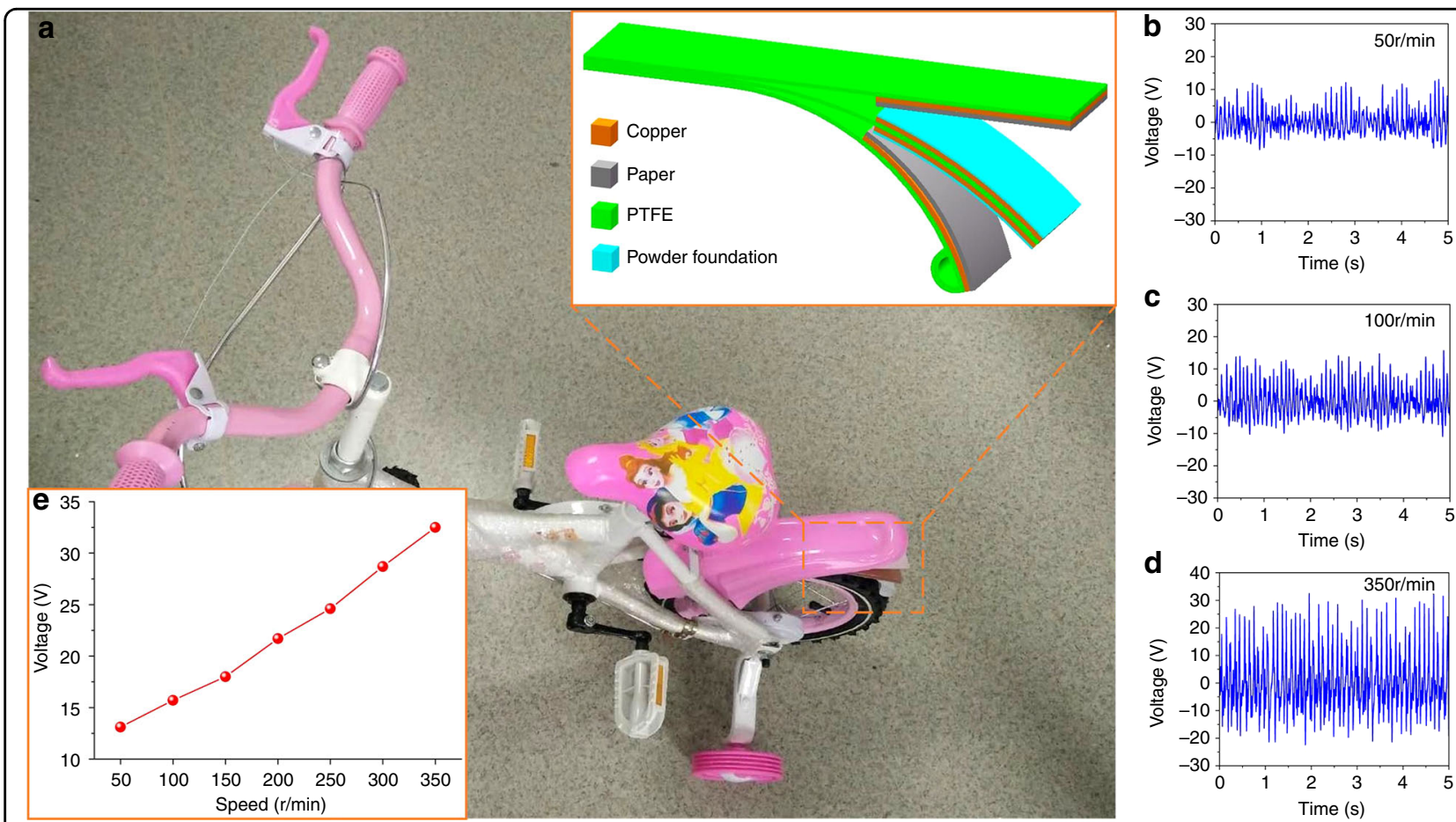

Fig. 8 Harvesting mechanical power associated with bicycle movement. a Photograph of a bicycle with a TENG (inset shows the palette structure of the TENG); b-d output voltage signals corresponding to three movement states (50, 100, and 350 r.p.m.). e The output voltages subjected to varied speeds 
a
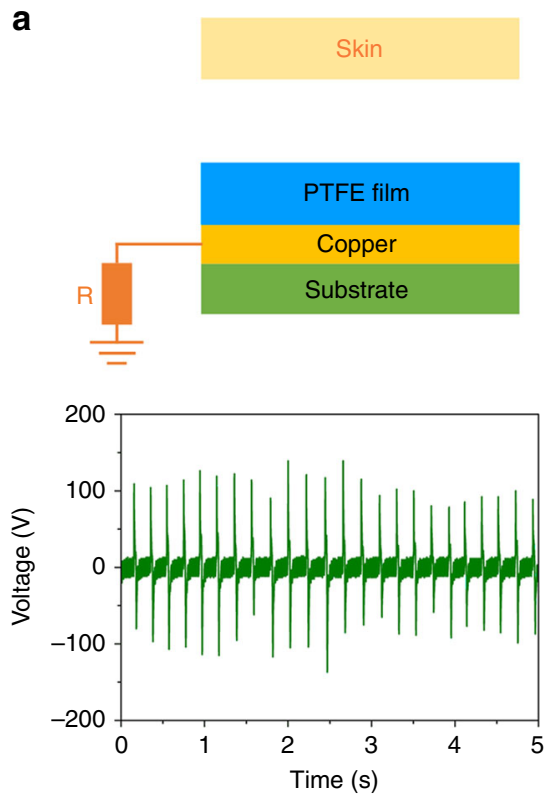

b
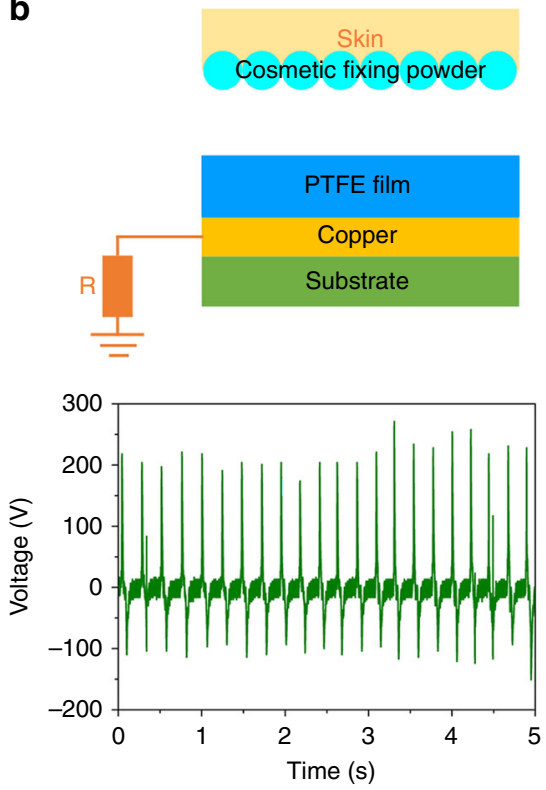

Fig. 9 Powder-enhanced skin-based TENG. Schematic illustration of $\mathbf{a}$ a skin-based TENG and $\mathbf{b}$ a cosmetic fixing powder-enhanced skin-based TENG and the corresponding output performance

\section{Conclusions}

A novel concept of powder electronics that use powder particulates (a cosmetic fixing powder) as triboelectric materials for fabricating a TENG is proposed in this work. The loose powder and Teflon tape are used as the triboelectric pair. The CFP-TENG can generate approximate $V_{\mathrm{OC}}, I_{\mathrm{SC}}$, and maximum power density values of $1141 \mathrm{~V}$, $521 \mu \mathrm{A}$, and $570.96 \mu \mathrm{W} / \mathrm{cm}^{2}$, respectively. Stable output voltage from the CFP-TENG is realized after a 3000-cycle external force test. In addition, this TENG, which was designed as a novel palette structure for harvesting mechanical energy from bicycle motion, serves as a selfpowered speed sensor. Furthermore, the cosmetic fixing powder can be used as an effective material to enhance the triboelectric property of skin. This work represents a significant step towards the large-scale production of TENGs.

\section{Materials and methods}

All materials are commercially available and used without further processing.

\section{Acknowledgements}

This work was supported by the National Natural Science Foundation of China (Grant No. 61804132, Grant No. 61674128, Grant No. 61731019) and the Aeronautical Science Foundation of China (Grant No. ASFC-2017ZC76002). The author would also like to thank Prof. Yufeng Jin and Prof. Min Yu from Peking University for their previous guidance.

\section{Authors' contributions}

K.X. performed the experiment and wrote the paper. Z.Z. analysed the data and wrote the paper. Y.C., J.F., H.Z., C.D., and Z.X. analysed the data.
Conflict of interest

The authors declare that they have no conflict of interest.

Supplementary information accompanies this paper at https://doi.org/ 10.1038/s41378-019-0066-1.

Received: 28 January 2019 Revised: 22 March 2019 Accepted: 27 March 2019

Published online: 01 July 2019

\section{References}

1. Ren, $X$. et al. Wind energy harvester based on coaxial rotatory freestanding triboelectric nanogenerators for self-powered water splitting. Nano Energy 50, 562-570 (2018).

2. Cao, X. et al. Inductor-free wireless energy delivery via Maxwell's displacement current from an electrodeless triboelectric nanogenerator. Adv. Mater. 30, 1704077 (2018)

3. Qian, Y., Du, J. \& Kang, D. J. Enhanced electrochemical performance of porous Co-doped $\mathrm{TiO}_{2}$ nanomaterials prepared by a solvothermal method. Microporous Mesoporous Mater. 273, 148-155 (2019).

4. Wu, C. et al. Keystroke dynamics enabled authentication and identification using triboelectric nanogenerator array. Mater. Today 21, 216-222 (2018).

5. Chen, $X$. et al. Hybrid generator based on freestanding magnet as all-direction in-plane energy harvester and vibration sensor. Nano Energy 49, 51-58 (2018).

6. Meng, K. et al. Flexible weaving constructed self-powered pressure sensor enabling continuous diagnosis of cardiovascular disease and measurement of Cuffless blood pressure. Adv. Funct. Mater. 5, 1806388 (2019).

7. Wang, Y. et al. Comprehensive pyro-phototronic effect enhanced ultraviolet detector with ZnO/Ag Schottky junction. Adv. Funct. Mater. 29, 1807111 (2018).

8. Chen, J. et al. Bladeless-turbine-based triboelectric nanogenerator for fluid energy harvesting and self-powered fluid gauge. Adv. Mater. Technol. 4, 1800560 (2018).

9. $\mathrm{Pu}, \mathrm{X}$. et al. Rotation sensing and gesture control of a robot joint via triboelectric quantization sensor. Nano Energy 54, 453-460 (2018).

10. $\mathrm{Wu}, \mathrm{H}$. et al. Self-powered noncontact electronic skin for motion sensing. $A d v$. Funct. Mater. 28, 1704641 (2018).

11. $\mathrm{Wu}, \mathrm{H}$. et al. Fabric-based self-powered noncontact smart gloves for gesture recognition. J. Mater. Chem. A 6, 20277-20288 (2018). 
12. Su, Z. et al. Microsphere-assisted robust epidermal strain gauge for static and dynamic gesture recognition. Small 13, 1702108 (2017).

13. Ren, $X$. et al. Coaxial rotatory-freestanding triboelectric nanogenerator for effective energy scavenging from wind. Smart Mater. Struct. 27, 065016 (2018).

14. Wu, C. et al. Bioprinting: an assessment based on manufacturing readiness levels. Crit. Rev. Biotechnol. 37, 333-354 (2017).

15. Zhang, X. S. et al. High-performance triboelectric nanogenerator with enhanced energy density based on single-step fluorocarbon plasma treatment. Nano Energy 4, 123-131 (2014).

16. Lei, $\mathrm{S}$. et al. Microstructure, phase evolution and interfacial effects in a new $\mathrm{Zn}_{0.9} \mathrm{Mg}_{0.1} \mathrm{TiO}_{3}-\mathrm{ZnNb}_{2} \mathrm{O}_{6}$ ceramic system with greatly induced improvement in microwave dielectric properties. Scr. Mater. 146, 154-159 (2018).

17. Wang, $X$. et al. Fully packaged blue energy harvester by hybridizing a rolling triboelectric nanogenerator and an electromagnetic generator. ACS Nano 10, 11369-11376 (2016)

18. $L i, X$. et al. 3D fiber-based hybrid nanogenerator for energy havesting and as a self-powered pressure sensor. ACS Nano 8, 10674-10681 (2014).

19. Chen, H. et al. Self-powered electronic skin based on the triboelectric generator. Nano Energy 56, 252-268 (2019).

20. Song, Y. et al. High-efficiency self-charging smart bracelet for portable electronics. Nano Energy 55, 29-36 (2019).

21. $\mathrm{Wu}, \mathrm{C}$. et al. Triboelectric nanogenerator: a foundation of the energy for the new era. Adv. Energy Mater. 9, 1802906 (2019).

22. Guo, H. et al. A highly sensitive, self-powered triboelectric auditory sensor for social robotics and hearing aids. Sci. Robot. 3, eaat2516 (2018).

23. Yang, J. et al. Broadband vibrational energy harvesting based on a triboelectric nanogenerator. Adv. Energy Mater. 4, 1301322 (2014).

24. Song, Y. et al. All-in-one piezoresistive-sensing patch integrated with microsupercapacitor. Nano Energy 53, 189-197 (2018).

25. Zhang, X. S. et al. High performance triboelectric nanogenerators based on large-scale mass-fabrication technologies. Nano Energy 11, 304-322 (2015).

26. Li, Z. et al. Multilayered fiber-based triboelectric nanogenerator with high performance for biomechanical energy harvesting. Nano Energy $\mathbf{5 3}, 726-733$ (2018).

27. Zhang, X. S., Brugger, J. \& Kim, B. A silk-fibroin-based transparent triboelectric generator suitable for autonomous sensor network. Nano Energy 20, 37-47 (2016).

28. Li, Z. et al. Nanofibrous membrane constructed wearable triboelectric nanogenerator for high performance biomechanical energy harvesting. Nano Energy 36, 341-348 (2017).
29. Yin, X. et al. Structure and dimension effects on the performance of layered triboelectric nanogenerators in contact-separation mode. ACS Nano, https:// doi.org/10.1021/acsnano.8b07935(2018).

30. Wei, G. et al. Self-powered hybrid flexible nanogenerator and its application in bionic micro aerial vehicles. Nano Energy 54, 10-16 (2018).

31. Tang, W. et al. Implantable self-powered low-level laser cure system for mouse embryonic osteoblasts' proliferation and differentiation. ACS Nano 9, 7867-7873 (2015)

32. Zhang, X. S. et al. Penciling a triboelectric nanogenerator on paper for autonomous power MEMS applications. Nano Energy 33, 393-401 (2017).

33. Tang, W. et al. Liquid-metal electrode for high-performance triboelectric nanogenerator at an instantaneous energy conversion efficiency of $70.6 \%$. Adv. Funct. Mater. 25, 3718-3725 (2015).

34. Zhang, X. S. et al. Frequency-multiplication high-output triboelectric nanogenerator for sustainably powering biomedical microsystems. Nano Lett. 13, 1168-1172 (2013).

35. Chen, $\mathrm{H}$. et al. Hybrid porous micro structured finger skin inspired selfpowered electronic skin system for pressure sensing and sliding detection. Nano Energy 51, 496-503 (2018).

36. Cheng, P. et al. Largely enhanced triboelectric nanogenerator for efficient harvesting of water wave energy by soft contacted structure. Nano Energy 57, 432-439 (2019).

37. Zhao, C. et al. Hybrid piezo/triboelectric nanogenerator for highly dfficient and stable rotation energy harvesting. Nano Energy 57, 440-449 (2019).

38. Chen, $\mathrm{H}$. et al. Fingertip-inspired electronic skin based on triboelectric sliding sensing and porous piezoresistive pressure detection. Nano Energy 40, 65-72 (2017).

39. Qian, Y. \& Kang, D. J. Polydimethylsiloxane/ZnO nanoflakes/Three-dimensional graphene heterostructures for high-performance flexible energy harvesters with simultaneous piezoelectric and triboelectric generation. ACS Appl. Mater. Interfaces 10, 32281-32288 (2018).

40. Guo, Y. et al. All-fiber hybrid piezoelectric-enhanced triboelectric nanogenerator for wearable gesture monitoring. Nano Energy 48, 152-160 (2018).

41. Qian, Y. \& Kang, D. J. Large-area high-quality AB-stacked bilayer graphene on h-BN/Pt foil by chemical vapor deposition. ACS Appl. Mater. Interfaces 10, 29069-29075 (2018).

42. Kim, I. et al. A triboelectric nanogenerator using silica-based powder for appropriate technology. Sens. Actuators A Phys. 280, 85-91 (2018).

43. Zhang, X. S. et al. All-in-one self-powered flexible microsystems based on triboelectric nanogenerators. Nano Energy 47, 410-426 (2018). 